\title{
On Refined Neutrosophic Vector Spaces II
}

\author{
${ }^{1}$ M.A. Ibrahim, ${ }^{2}$ A.A.A. Agboola,${ }^{3}$ B.S. Badmus, ${ }^{4}$ S.A. Akinleye \\ ${ }^{1,2,4}$ Department of Mathematics, Federal University of Agriculture, Abeokuta, Nigeria. \\ ${ }^{3}$ Department of Physics, Federal University of Agriculture, Abeokuta, Nigeria. \\ muritalaibrahim40@gmail.com ${ }^{1}$, agboolaaaa@funaab.edu.ng ${ }^{2}$, badmusbs@yahoo.com ${ }^{3}$, \\ sa_akinleye@yahoo.com ${ }^{4}$
}

\begin{abstract}
The concept of refined neutrosophic vector spaces was introduced by Ibrahim et al. in [20] and the present paper is the continuation of the work. In the present paper, further studies on neutrosophic vector spaces are presented. Specifically, linear dependence, independence, bases and dimensions of refined neutrosophic vector spaces are studied with several results and examples presented. Also, refined neutrosophic homomorphisms of refined neutrosophic vector spaces are studied and existence of linear maps between weak refined neutrosophic vector spaces and weak neutrosophic vector spaces are established.
\end{abstract}

Keywords: Neutrosophy, neutrosophic vector space, refined neutrosophic vector space, refined neutrosophic vector space homomorphism.

\section{Introduction and Preliminaries}

Neutrosophy is a new branch of philosophy introduced by Florentin Smarandache in 1995. Neutrosophic logic/set introduced by Smarandache in [28] is an extension of fuzzy logic/set introduced by Zadeh [38] and intuitionistic fuzzy logic/set introduced by Atanassov [13]. In neutrosophic logic/set, each proposition is characterized by the degree of truth in the set $(T)$, degree if indeterminacy in the set $(I)$ and the degree of falsehood in the set $(F)$ where $(T, I, F)$ are not necessarily intervals, but may be any real sub-unitary subsets: discrete or continuous; single-element, finite, or (countable or uncountable) infinite; union or intersection of various subsets; etc. Neutrosophic logic/set has many applications in mathematics, computer science, engineering, technology, decision making, medical diagnosis, social sciences and many other fields. For full details, the

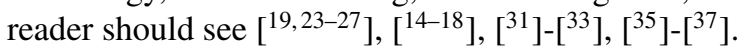

Smarandache recently introduced the concept of refined neutrosophic logic/set in [29] where it was shown that the neutrosophic components $(T, I, F)$ can be split into refined neutrosophic components of the form $<T_{1}, T_{2}, \cdots, T_{p} ; I_{1}, I_{2}, \cdot, I_{r} ; F_{1}, F_{2}, \cdots, F_{s}>$ with applications in physics and other sciences and mathematics. In [30], Smarandache presented $(T, I, F)$ structures and this motivated Agboola to introduce the concept of refined neutrosophic algebraic structures in ${ }^{8}$ where he studied refined neutrosophic groups. Since the introduction of refined neutrosophic algebraic structures, many researchers have further studied the concepts

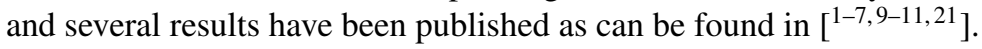

The concept of a neutrosophic vector space $V(I)$ generated by a vector space $V$ and indeterminacy factor $I$ was introduced by Vasantha Kandasamy and Florentin Smarandache in [31]. Since then, several researchers have studied the concept and a great deal of literature have been published. Recently, Agboola and Akinleye in [12] studied classical vector spaces in a neutrosophic environment and they showed that every neutrosophic vector space over a neutrosophic field (resp. field) is a vector space. In [ [34], Vasantha Kandasamy, et al. introduced for the first time the concept of neutrosophic quadruple vector spaces over the classical fields $\mathbb{R}, \mathbb{C}$ and $\mathbb{Z}_{p}$ and they presented several interesting results. Further studies on neutrosophic quadruple vector spaces were carried out in [22] by Ibrahim et al. where several results and examples were presented. The notion of refined neutrosophic vector spaces and their properties was introduces by Ibrahim et al. in [20]. They studied Weak(strong) refined neutrosophic vector spaces and subspaces, and also, they studied strong refined 
neutrosophic quotient vector spaces. Several interesting results and examples were presented. It was shown that every weak (strong) refined neutrosophic vector space is a vector space and it was equally shown that every strong refined neutrosophic vector space is a weak refined neutrosophic vector space. In the present paper however, further studies on refined neutrosophic vector spaces are presented. Specifically, linear dependence, independence, bases and dimensions of refined neutrosophic vector spaces are studied and several results and examples are presented. Refined neutrosophic homomorphisms of refined vector spaces are studied and existence of linear maps between weak refined neutrosophic vector spaces $V\left(I_{1}, I_{2}\right)$ and weak neutrosophic vector spaces $V(I)$ are established.

For the purposes of this paper, it will be assumed that I splits into two indeterminacies $I_{1}$ [contradiction (true $(T)$ and false $(F))$ ] and $I_{2}$ [ignorance (true $(T)$ or false $(F)$ )]. It then follows logically that:

$$
\begin{aligned}
& I_{1} I_{1}=I_{1}^{2}=I_{1}, \\
& I_{2} I_{2}=I_{2}^{2}=I_{2}, \text { and } \\
& I_{1} I_{2}=I_{2} I_{1}=I_{1} .
\end{aligned}
$$

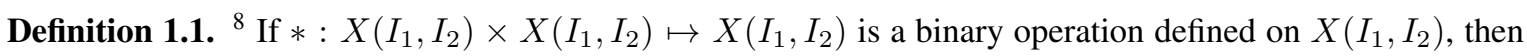
the couple $\left(X\left(I_{1}, I_{2}\right), *\right)$ is called a refined neutrosophic algebraic structure and it is named according to the laws (axioms) satisfied by $*$.

Definition 1.2. ${ }^{8}$ Let $\left(X\left(I_{1}, I_{2}\right),+,.\right)$ be any refined neutrosophic algebraic structure where + and . are ordinary addition and multiplication respectively.

For any two elements $\left(a, b I_{1}, c I_{2}\right),\left(d, e I_{1}, f I_{2}\right) \in X\left(I_{1}, I_{2}\right)$, we define

$$
\begin{gathered}
\left(a, b I_{1}, c I_{2}\right)+\left(d, e I_{1}, f I_{2}\right)=\left(a+d,(b+e) I_{1},(c+f) I_{2}\right), \\
\left(a, b I_{1}, c I_{2}\right) \cdot\left(d, e I_{1}, f I_{2}\right)=\left(a d,(a e+b d+b e+b f+c e) I_{1},(a f+c d+c f) I_{2}\right) .
\end{gathered}
$$

Definition 1.3. $\frac{8}{\text { If }}{ }^{\prime \prime}+{ }^{\prime \prime}$ and $" . "$ are ordinary addition and multiplication, $I_{k}$ with $k=1,2$ have the following properties:

1. $I_{k}+I_{k}+\cdots+I_{k}=n I_{k}$.

2. $I_{k}+\left(-I_{k}\right)=0$.

3. $I_{k} \cdot I_{k} \cdots I_{k}=I_{k}^{n}=I_{k}$ for all positive integers $n>1$.

4. $0 \cdot I_{k}=0$.

5. $I_{k}^{-1}$ is undefined and therefore does not exist.

Definition 1.4. ${ }^{8}$ Let $(G, *)$ be any group. The couple $\left(G\left(I_{1}, I_{2}\right), *\right)$ is called a refined neutrosophic group generated by $G, I_{1}$ and $I_{2}$. $\left(G\left(I_{1}, I_{2}\right), *\right)$ is said to be commutative if for all $x, y \in G\left(I_{1}, I_{2}\right)$, we have $x * y=y * x$. Otherwise, we call $\left(G\left(I_{1}, I_{2}\right), *\right)$ a non -commutative refined neutrosophic group.

Definition 1.5. $\frac{8}{\text { If }}\left(X\left(I_{1}, I_{2}\right), *\right)$ and $\left(Y\left(I_{1}, I_{2}\right), *^{\prime}\right)$ are two refined neutrosophic algebraic structures, the mapping

$$
\phi:\left(X\left(I_{1}, I_{2}\right), *\right) \longrightarrow\left(Y\left(I_{1}, I_{2}\right), *^{\prime}\right)
$$

is called a neutrosophic homomorphism if the following conditions hold:

1. $\phi\left(\left(a, b I_{1}, c I_{2}\right) *\left(d, e I_{1}, f I_{2}\right)\right)=\phi\left(\left(a, b I_{1}, c I_{2}\right)\right) *^{\prime} \phi\left(\left(d, e I_{1}, f I_{2}\right)\right)$.

2. $\phi\left(I_{k}\right)=I_{k}$ for all $\left(a, b I_{1}, c I_{2}\right),\left(d, e I_{1}, f I_{2}\right) \in X\left(I_{1}, I_{2}\right)$ and $k=1,2$.

Example 1.6. ${ }^{8}$ Let

$\mathbb{Z}_{2}\left(I_{1}, I_{2}\right)=\left\{(0,0,0),(1,0,0),\left(0, I_{1}, 0\right),\left(0,0, I_{2}\right),\left(0, I_{1}, I_{2}\right),\left(1, I_{1}, 0\right),\left(1,0, I_{2}\right),\left(1, I_{1}, I_{2}\right)\right\}$.

Then $\left(\mathbb{Z}_{2}\left(I_{1}, I_{2}\right),+\right)$ is a commutative refined neutrosophic group of integers modulo 2 .

Generally for a positive integer $n \geq 2,\left(\mathbb{Z}_{n}\left(I_{1}, I_{2}\right),+\right)$ is a finite commutative refined neutrosophic group of integers modulo $n$.

Example 1.7. ${ }^{8}$ Let $\left(G\left(I_{1}, I_{2}\right), *\right)$ and and $\left(H\left(I_{1}, I_{2}\right), *^{\prime}\right)$ be two refined neutrosophic groups.

Let $\phi: G\left(I_{1}, I_{2}\right) \times H\left(I_{1}, I_{2}\right) \rightarrow G\left(I_{1}, I_{2}\right)$ be a mapping defined by $\phi(x, y)=x$ and let

$\psi: G\left(I_{1}, I_{2}\right) \times H\left(I_{1}, I_{2}\right) \rightarrow H\left(I_{1}, I_{2}\right)$ be a mapping defined by $\psi(x, y)=y$. Then $\phi$ and $\psi$ are refined neutrosophic group homomorphisms. 
Definition 1.8. ${ }^{4}$ Let $(R,+,$.$) be any ring. The abstract system \left(R\left(I_{1}, I_{2}\right),+,.\right)$ is called a refined neutrosophic ring generated by $R, I_{1}, I_{2}$. $\left(R\left(I_{1}, I_{2}\right),+,.\right)$ is called a commutative refined neutrosophic ring if for all $x, y \in R\left(I_{1}, I_{2}\right)$, we have $x y=y x$. If there exists an element $e=(1,0,0) \in R\left(I_{1}, I_{2}\right)$ such that $e x=x e=x$ for all $x \in R\left(I_{1}, I_{2}\right)$, then we say that $\left(R\left(I_{1}, I_{2}\right),+,.\right)$ is a refined neutrosophic ring with unity.

Definition 1.9. ${ }^{4}$ Let $\left(R\left(I_{1}, I_{2}\right),+,.\right)$ be a refined neutrosophic ring and let $n \in \mathbb{Z}^{+}$.

(i) If $n x=0$ for all $x \in R\left(I_{1}, I_{2}\right)$, we call $\left(R\left(I_{1}, I_{2}\right),+,.\right)$ a refined neutrosophic ring of characteristic $n$ and $n$ is called the characteristic of $\left(R\left(I_{1}, I_{2}\right),+,.\right)$.

(ii) $\left(R\left(I_{1}, I_{2}\right),+,.\right)$ is call a refined neutrosophic ring of characteristic zero if for all $x \in R\left(I_{1}, I_{2}\right), n x=0$ is possible only if $n=0$.

\section{Example 1.10. 4}

(i) $\mathbb{Z}\left(I_{1}, I_{2}\right), \mathbb{Q}\left(I_{1}, I_{2}\right), \mathbb{R}\left(I_{1}, I_{2}\right), \mathbb{C}\left(I_{1}, I_{2}\right)$ are commutative refined neutrosophic rings with unity of characteristics zero.

(ii) Let $\mathbb{Z}_{2}\left(I_{1}, I_{2}\right)=\left\{(0,0,0),(1,0,0),\left(0, I_{1}, 0\right),\left(0,0, I_{2}\right),\left(0, I_{1}, I_{2}\right),\left(1, I_{1}, 0\right),\left(1,0, I_{2}\right),\left(1, I_{1}, I_{2}\right)\right\}$. Then $\left(\mathbb{Z}_{2}\left(I_{1}, I_{2}\right),+,.\right)$ is a commutative refined neutrosophic ring of integers modulo 2 of characteristic 2. Generally for a positive integer $n \geq 2,\left(\mathbb{Z}_{n}\left(I_{1}, I_{2}\right),+,.\right)$ is a finite commutative refined neutrosophic ring of integers modulo $n$ of characteristic $n$.

Example 1.11. ${ }^{4}$ Let $M_{n \times n}^{\mathbb{R}}\left(I_{1}, I_{2}\right)=\left\{\left[\begin{array}{llll}a_{11} & a_{12} & \cdots & a_{1 n} \\ a_{21} & a_{22} & \cdots & a_{2 n} \\ \vdots & \vdots & \vdots & \vdots \\ a_{n 1} & a_{n 2} & \cdots & a_{n n}\end{array}\right]: a_{i j} \in \mathbb{R}\left(I_{1}, I_{2}\right)\right\}$

be a refined neutrosophic set of all $n \times n$ matrix. Then $\left(M_{n \times n}^{\mathbb{R}}\left(I_{1}, I_{2}\right),+,.\right)$ is a non-commutative refined neutrosophic ring under matrix multiplication.

Theorem 1.12. ${ }^{4}$ Let $\left(R\left(I_{1}, I_{2}\right),+,.\right)$ be any refined neutrosophic ring. Then $\left(R\left(I_{1}, I_{2}\right),+,.\right)$ is a ring.

\section{Linear dependence, independence, bases and dimensions of a refined neutrosophic vector space}

Definition 2.1. Let $(V,+,$.$) be any vector space over a field \mathrm{K}$. Let $V\left(I_{1}, I_{2}\right)=<V \cup\left(I_{1}, I_{2}\right)>$ be a refined neutrosophic set generated by $V, I_{1}$ and $I_{2}$. We call the triple $\left(V\left(I_{1}, I_{2}\right),+,.\right)$ a weak refined neutrosophic vector space over a field $\mathrm{K}$, if $V\left(I_{1}, I_{2}\right)$ is a refined neutrosophic vector space over a refined neutrosophic field $K\left(I_{1}, I_{2}\right)$, then $V\left(I_{1}, I_{2}\right)$ is called a strong refined neutrosophic vector space.

The elements of $V\left(I_{1} I_{2}\right)$ are called refined neutrosophic vectors and the elements of $K\left(I_{1}, I_{2}\right)$ are called refined neutrosophic scalars.

If $u=a+b I_{1}+c I_{2}, v=d+e I_{1}+f I_{2} \in V\left(I_{1}, I_{2}\right)$ where a, b, c, d, e and $\mathrm{f}$ are vectors in $\mathrm{V}$ and $\alpha=k+m I_{1}+n I_{2} \in K\left(I_{1}, I_{2}\right)$ where $k, m$ and $n$ are scalars in $K$, we define:

$$
u+v=\left(a+b I_{1}+c I_{2}\right)+\left(d+e I_{1}+f I_{2}\right)=(a+d)+(b+e) I_{1}+(c+f) I_{2},
$$

and

$\alpha u=\left(k+m I_{1}+n I_{2}\right) \cdot\left(a+b I_{1}+c I_{2}\right)=k . a+(k . b+m . a+m . b+m . c+n . b) I_{1}+(k . c+n . a+n . c) I_{2}$.

Definition 2.2. Let $V\left(I_{1}, I_{2}\right)$ be a strong refined neutrosophic vector space over a refined neutrosophic field $K\left(I_{1}, I_{2}\right)$ and let $v_{1}, v_{2}, \cdot, v_{n} \in V\left(I_{1}, I_{2}\right)$.

1. An element $v \in V\left(I_{1}, I_{2}\right)$ is said to be a linear combination of the $v_{i}^{\prime} s$ if

$$
v=\alpha_{1} v_{1}+\alpha_{2} v_{2}+\cdots+\alpha_{n} v_{n}, \text { where } \quad \alpha_{i} \in K\left(I_{1}, I_{2}\right) .
$$


2. $v_{i}^{\prime} s$ are said to be linearly independent if

$$
\alpha_{1} v_{1}+\alpha_{2} v_{2}+\cdots+\alpha_{n} v_{n}=0
$$

implies that $\alpha_{1}=\alpha_{2}=\cdots=\alpha_{n}=0$.

In this case, the set $\left\{v_{1}, v_{2}, \cdots, v_{n}\right\}$ is called a linearly independent set.

3. $v_{i}^{\prime} s$ are said to be linearly dependent if

$$
\alpha_{1} v_{1}+\alpha_{2} v_{2}+\cdots+\alpha_{n} v_{n}=0
$$

implies that not all $\alpha_{i}$ are equal to zero.

In this case, the set $\left\{v_{1}, v_{2}, \cdots, v_{n}\right\}$ is called a linearly dependent set.

Definition 2.3. Let $V\left(I_{1}, I_{2}\right)$ be a weak refined neutrosophic vector space over a field $K$ and let $v_{1}, v_{2}, \cdots, v_{n} \in V\left(I_{1}, I_{2}\right)$.

1. An element $v \in V\left(I_{1}, I_{2}\right)$ is said to be a linear combination of the $v_{i}^{\prime} s$ if

$$
v=k_{1} v_{1}+k_{2} v_{2}+\cdots+k_{n} v_{n}, \text { where } \quad k_{i} \in K .
$$

2. $v_{i}^{\prime} s$ are said to be linearly independent if

$$
k_{1} v_{1}+k_{2} v_{2}+\cdots+k_{n} v_{n}=0
$$

implies that $k_{1}=k_{2}=\cdots=k_{n}=0$.

In this case, the set $\left\{v_{1}, v_{2}, \cdots, v_{n}\right\}$. is called a linearly independent set.

3. $v_{i}^{\prime} s$ are said to be linearly dependent if

$$
k_{1} v_{1}+k_{2} v_{2}+\cdots+k_{n} v_{n}=0 .
$$

implies that not all $k_{i}$ are equal to zero.

In this case, the set $\left\{v_{1}, v_{2}, \cdots, v_{n}\right\}$ is called a linearly dependent set.

Example 2.4. Let $V\left(I_{1}, I_{2}\right)=\mathbb{R}\left(I_{1}, I_{2}\right)$ be a weak refined neutrosophic vector space over a field $K=\mathbb{R}$. An element $v=8+19 I_{1}+18 I_{2} \in V\left(I_{1}, I_{2}\right)$ is a linear combination of the elements $v_{1}=2+5 I_{1}+4 I_{2}$, $v_{2}=1+2 I_{1}+3 I_{2} \in V\left(I_{1}, I_{2}\right)$, since $8+19 I_{1}+18 I_{2}=3\left(2+5 I_{1}+4 I_{2}\right)+2\left(1+2 I_{1}+3 I_{2}\right)$.

Example 2.5. Let $V\left(I_{1}, I_{2}\right)=\mathbb{R}\left(I_{1}, I_{2}\right)$ be a weak refined neutrosophic vector space over a field $K=\mathbb{R}$. An element $v=3+15 I_{1}+7 I_{2} \in V\left(I_{1}, I_{2}\right)$ is a linear combination of the elements $v_{1}=2+5 I_{1}+3 I_{2}$, $v_{2}=1+I_{1}+I_{2} \in V\left(I_{1}, I_{2}\right)$, since $3+15 I_{1}+7 I_{2}=4\left(2+5 I_{1}+3 I_{2}\right)-5\left(1+I_{1}+I_{2}\right)$.

Example 2.6. Let $V\left(I_{1}, I_{2}\right)=R\left(I_{1}, I_{2}\right)$ be a strong refined neutrosophic vector space over a refined neutrosophic field $K\left(I_{1}, I_{2}\right)=\mathbb{R}\left(I_{1}, I_{2}\right)$. An element $v=8+19 I_{1}+18 I_{2} \in V\left(I_{1}, I_{2}\right)$ is a linear combination of the elements $v_{1}=1+2 I_{1}+3 I_{2}, v_{2}=2+5 I_{1}+4 I_{2} \in V\left(I_{1}, I_{2}\right)$, since

$$
\begin{aligned}
8+19 I_{1}+18 I_{2} & =\left(2+5 I_{1}+6 I_{2}\right)\left(1+2 I_{1}+3 I_{2}\right)+\left(3-2 I_{1}-4 I_{2}\right)\left(2+5 I_{1}+4 I_{2}\right) \\
& =\left(2+8 I_{1}+3 I_{2}\right)\left(1+2 I_{1}+3 I_{2}\right)+\left(3-4 I_{1}-2 I_{2}\right)\left(2+5 I_{1}+4 I_{2}\right) \\
& =\left(4+11 I_{1}-2 I_{2}\right)\left(1+2 I_{1}+3 I_{2}\right)+\left(2-6 I_{1}+I_{2}\right)\left(2+5 I_{1}+4 I_{2}\right) \\
& =\left(4+8 I_{1}+I_{2}\right)\left(1+2 I_{1}+3 I_{2}\right)+\left(2-4 I_{1}-I_{2}\right)\left(2+5 I_{1}+4 I_{2}\right) .
\end{aligned}
$$

Here $\left(2+5 I_{1}+6 I_{2}\right),\left(3-2 I_{1}-4 I_{2}\right),\left(2+8 I_{1}+3 I_{2}\right),\left(3-4 I_{1}-2 I_{2}\right),\left(4+11 I_{1}-2 I_{2}\right)$, $\left(2-6 I_{1}+I_{2}\right),\left(4+8 I_{1}+I_{2}\right),\left(2-4 I_{1}-I_{2}\right) \in K\left(I_{1}, I_{2}\right)$.

This example shows that the element $v=8+19 I_{1}+18 I_{2}$ can be infinitely expressed as a linear combination of the elements $v_{1}=1+2 I_{1}+3 I_{2}, v_{2}=2+5 I_{1}+4 I_{2} \in V\left(I_{1}, I_{2}\right)$. This observation is recorded in the next proposition.

Proposition 2.7. Let $V\left(I_{1}, I_{2}\right)$ be a strong refined neutrosophic vector space over a neutrosophic field $K\left(I_{1}, I_{2}\right)$ and let $v_{1}, v_{2}, \cdots, v_{n} \in V\left(I_{1}, I_{2}\right)$. An element $v \in V\left(I_{1}, I_{2}\right)$ can be infinitely expressed as a linear combination of the $v_{i} s$. 
Proof: Suppose that $v=\alpha_{1} v_{1}+\alpha_{2} v_{2}+\cdots+\alpha_{n} v_{n}$ where $v=a+b I_{1}+c I_{2}, v_{1}=a_{1}+b_{1} I_{1}+c_{1} I_{2}$, $v_{2}=a_{2}+b_{2} I_{1}+c I_{2}, \cdots, v_{n}=a_{n}+b_{n} I_{1}+c I_{n}$ and $\alpha_{1}=k_{1}+m_{1} I_{1}+t I_{2}, \alpha_{2}=k_{2}+m_{2} I_{1}+t I_{2}, \cdots, \alpha_{n}=$ $k_{n}+m_{n} I_{1}+t I_{2} \in K\left(I_{1}, I_{2}\right)$.

Then

$a+b I_{1}+c I_{2}=\left(k_{1}+m_{1} I_{1}+t_{1} I_{2}\right)\left(a_{1}+b_{1} I_{1}+c_{1} I_{2}\right)+\left(k_{2}+m_{2} I_{1}+t_{2} I_{2}\right)\left(a_{2}+b_{2} I_{1}+c_{2} I_{2}\right)+\cdots+$ $\left(k_{n}+m_{n} I_{1}+t_{n} I_{2}\right)\left(a_{n}+b_{n} I_{1}+c_{n} I_{2}\right)$

from which we obtain

$a_{1} k_{1}+a_{2} k_{2}+\cdots+a_{n} k_{n}=a$

$b_{1} k_{1}+a_{1} m_{1}+b_{1} m_{1}+c_{1} m_{1}+b_{1} t_{1}+b_{2} k_{2}+a_{2} m_{2}+b_{2} m_{2}+c_{2} m_{2}+b_{2} t_{2}+\cdots+b_{n} k_{n}+a_{n} m_{n}+b_{n} m_{n}+$ $c_{n} m_{n}+b_{n} t_{n}=b$

$c_{1} k_{1}+a_{1} t_{1}+c_{1} t_{1}+c_{2} k_{2}+a_{2} t_{2}+c_{2} t_{2}+\cdots+c_{n} k_{n}+a_{n} t_{n}+c_{n} t_{n}=c$.

This is a linear system in unknowns $k_{i}, m_{i}, t_{i} \quad i=1,2, \cdots, n$.

Since the system is consistent and have infinitely many solutions, it follows that the $v_{i} s$ can be infinitely combined to produce $\mathrm{v}$.

But if $V\left(I_{1}, I_{2}\right)$ and $K\left(I_{1}, I_{2}\right)$ are finite the $v_{i} s$ will be finitely combined to produce $\mathrm{v}$.

Proposition 2.8. Let $V\left(I_{1}, I_{2}\right)$ be a strong refined neutrosophic vector space over a refined neutrosophic field $K\left(I_{1}, I_{2}\right)$ and let $U\left[I_{1}, I_{2}\right]$ and $W\left[I_{1}, I_{2}\right]$ be subsets of $V\left[I_{1}, I_{2}\right]$ such that $U\left[I_{1}, I_{2}\right] \subseteq W\left[I_{1}, I_{2}\right]$. If $U\left[I_{1}, I_{2}\right]$ is linearly dependent, then $W\left[I_{1}, I_{2}\right]$ is linearly dependent.

Proposition 2.9. Let $V\left(I_{1}, I_{2}\right)$ be a strong refined neutrosophic vector space over a refined neutrosophic field $K\left(I_{1}, I_{2}\right)$ and let $U\left[I_{1}, I_{2}\right]$ and $W\left[I_{1}, I_{2}\right]$ be subsets of $V\left[I_{1}, I_{2}\right]$ such that $U\left[I_{1}, I_{2}\right] \subseteq W\left[I_{1}, I_{2}\right]$. If $W\left[I_{1}, I_{2}\right]$ is linearly independent, then $U\left[I_{1}, I_{2}\right]$ is linearly independent.

Proof : Let $W\left[I_{1}, I_{2}\right]=\left\{v_{1}=\left(a_{1}+b_{1} I_{1}+c_{1} I_{2}\right), v_{2}=\left(a_{2}+b_{2} I_{1}+c_{2} I_{2}\right), \cdots, v_{n}=\left(a_{n}+b_{n} I_{1}+c_{n} I_{2}\right)\right\}$, be a linearly independent set. Let, if possible,

$U\left[I_{1}, I_{2}\right]=\left\{v_{1}=\left(a_{1}+b_{1} I_{1}+c_{1} I_{2}\right), v_{2}=\left(a_{2}+b_{2} I_{1}+c_{2} I_{2}\right), \cdots, v_{z}=\left(a_{z}+b_{z} I_{1}+c_{z} I_{2}\right)\right\}, z<n$, be a linearly dependent subset of $\left\{v_{1}=\left(a_{1}+b_{1} I_{1}+c_{1} I_{2}\right), v_{2}=\left(a_{2}+b_{2} I_{1}+c_{2} I_{2}\right), \cdots, v_{n}=\left(a_{n}+b_{n} I_{1}+c_{n} I_{2}\right)\right\}$. Then there exist some scalars $\left(k_{1}+m_{1} I_{1}+t_{1} I_{2}\right),\left(k_{2}+m_{2} I_{1}+t_{2} I_{2}\right), \cdots\left(k_{z}+m_{z} I_{1}+t_{z} I_{2}\right) \in K\left(I_{1}, I_{2}\right)$, not all zero, such that

$\left(k_{1}+m_{1} I_{1}+t_{1} I_{2}\right)\left(a_{1}+b_{1} I_{1}+c_{1} I_{2}\right)+\left(k_{2}+m_{2} I_{1}+t_{2} I_{2}\right)\left(a_{2}+b_{2} I_{1}+c_{2} I_{2}\right)+\cdots+$

$\left(k_{z}+m_{z} I_{1}+t_{z} I_{2}\right)\left(a_{z}+b_{z} I_{1}+c_{z} I_{2}\right)=0$.

$\Longrightarrow\left(k_{1}+m_{1} I_{1}+t_{1} I_{2}\right)\left(a_{1}+b_{1} I_{1}+c_{1} I_{2}\right)+\left(k_{2}+m_{2} I_{1}+t_{2} I_{2}\right)\left(a_{2}+b_{2} I_{1}+c_{2} I_{2}\right)+\cdots+$

$\left(k_{z}+m_{z} I_{1}+t_{z} I_{2}\right)\left(a_{z}+b_{z} I_{1}+c_{z} I_{2}\right)+\left(0_{z+1}+0_{z+1} I_{1}+0_{z+1} I_{2}\right)\left(a_{z+1}+b_{z+1} I_{1}+c_{z+1} I_{2}\right)+$

$\left(0_{z+2}+0_{z+2} I_{1}+0_{z+2} I_{2}\right)\left(a_{z+2}+b_{z+2} I_{1}+c_{z+2} I_{2}\right)+\cdots+\left(0_{n}+0_{n} I_{1}+0_{n} I_{2}\right)\left(a_{n}+b_{n} I_{1}+c_{n} I_{2}\right)=0$.

The scalars $\left(k_{1}, m_{1} I_{1}, t_{1} I_{2}\right),\left(k_{2}, m_{2} I_{1}, t_{2} I_{2}\right), \cdots,\left(k_{z}, m_{z} I_{1}, t_{z} I_{2}\right),\left(0_{z+1}+0_{z+1} I_{1}+0_{z+1} I_{2}\right)$,

$\left(0_{z+2}+0_{z+2} I_{1}+0_{z+2} I_{2}\right), \cdots,\left(0_{n}+0_{n} I_{1}+0_{n} I_{2}\right)$ are not all zero.

Thus, the vectors $v_{1}=\left(a_{1}+b_{1} I_{1}+c_{1} I_{2}\right), v_{2}=\left(a_{2}+b_{2} I_{1}+c_{2} I_{2}\right), \cdots, v_{n}=\left(a_{n}+b_{n} I_{1}+c_{n} I_{2}\right)$ are linearly dependent. This contradiction the assumption that the vectors

$v_{1}=\left(a_{1}+b_{1} I_{1}+c_{1} I_{2}\right), v_{2}=\left(a_{2}+b_{2} I_{1}+c_{2} I_{2}\right), \cdots, v_{n}=\left(a_{n}+b_{n} I_{1}+c_{n} I_{2}\right)$ are linearly independent.

Hence, the set $\left\{v_{1}=\left(a_{1}+b_{1} I_{1}+c_{1} I_{2}\right), v_{2}=\left(a_{2}+b_{2} I_{1}+c_{2} I_{2}\right), \cdots, v_{z}=\left(a_{n}+b_{z} I_{1}+c_{z} I_{2}\right)\right\}$ is a linearly independent set.

Proposition 2.10. Let $V\left(I_{1}, I_{2}\right)$ be a weak refined neutrosophic vector space over a field $K$. The set $W\left(I_{1}, I_{2}\right)=\left\{v_{1}, v_{2}, \cdots v_{n}\right\} \subseteq V\left(I_{1}, I_{2}\right)$ is linearly dependent, if and only if at least one vector $v_{i}$ is a linear combination of the other vectors .

Proof : The proof is similar to the proof in classical case.

Proposition 2.11. Let $V\left(I_{1}, I_{2}\right)$ be a strong refined neutrosophic vector space over a neutrosophic field $K\left(I_{1}, I_{2}\right)$ and let $v_{1}=k_{1}+k_{1} I_{1}+k_{1} I_{2}, v_{2}=k_{2}+k_{2} I_{1}+k_{2} I_{2}, \cdots, v_{n}=k_{n}+k_{n} I_{1}+k_{n} I_{2}$ be elements of $V\left(I_{1}, I_{2}\right)$ where $0 \neq k_{i} \in K$. Then $\left\{v_{1}, v_{2}, \cdots, v_{n}\right\}$ is a linearly dependent set.

Proof :

Let $\alpha_{1}=p_{1}+q_{1} I_{1}+r_{1} I_{2}, \alpha_{2}=p_{2}+q_{2} I_{1}+r_{2} I_{2}, \cdots, \alpha_{n}=p_{n}+q_{n} I_{1}+r_{n} I_{2}$ be elements of $K\left(I_{1}, I_{2}\right)$. Then $\alpha_{1} v_{1}+\alpha_{2} v_{2}+\cdots+\alpha_{n} v_{n}=0$ which implies that

$\left(p_{1}+q_{1} I_{1}+r_{1} I_{2}\right)\left(k_{1}+k_{1} I_{1}+k_{2} I_{2}\right)+\left(p_{2}+q_{2} I_{1}+r_{2} I_{2}\right)\left(k_{2}+k_{2} I_{1}+k_{2} I_{2}\right)+\cdots+\left(p_{n}+q_{n} I_{1}+r_{n} I_{2}\right)\left(k_{n}+\right.$ $\left.k_{n} I_{1}+k_{n} I_{2}\right)=0$

from which we obtain

$k_{1} p_{1}+k_{2} p_{2}+\cdots+k_{n} p_{n}=0$. 
This is a homogeneous linear system in unknowns $p_{i}, i=1,2, \cdots, n$.

This system has infinitely many nontrivial solutions. Hence $\alpha_{i} s$ are not all zero and therefore, $\left\{v_{1}, v_{2}, \cdots, v_{n}\right\}$ is a linearly dependent set.

Note 1. If in Proposition 2.11 we consider a single vector $v \in V\left(I_{1}, I_{2}\right)$, the statement still hold. For instance, let $0 \neq v=a+b I_{1}-a I_{2} \in V\left(I_{1}, I_{2}\right)$ and $0 \neq \beta=p I_{1}-p I_{2} \in K\left(I_{1}, I_{2}\right)$, we have

$$
\beta \cdot v=\left(a+b I_{1}-a I_{2}\right) \cdot\left(p I_{1}-p I_{2}\right)=a p I_{1}+b p I_{1}-b p I_{1}-a p I_{1}-a p I_{2}+a p I_{2}=0 .
$$

Definition 2.12. Let $V\left(I_{1}, I_{2}\right)$ be weak(strong) refined neutrosophic vector space. If $\left\{v_{1}, v_{2}, \cdots, v_{n}\right\}$ is any set of refined neutrosophic vectors in $V\left(I_{1}, I_{2}\right)$, the set of all linear combinations of these refined neutrosophic vectors is called their span, and is denoted by

$$
\operatorname{span}\left\{v_{1}, v_{2}, \cdots, v_{n}\right\}
$$

If it happens that $V\left(I_{1}, I_{2}\right)=\operatorname{span}\left\{v_{1}, v_{2} \cdots, v_{n}\right\}$, then these vectors are called a spanning set for $V\left(I_{1}, I_{2}\right)$.

Proposition 2.13. Let $U\left(I_{1}, I_{2}\right)=\operatorname{span}\left\{v_{1}, v_{2}, \cdots, v_{n}\right\}$ be in a strong refined neutrosophic vector space $V\left(I_{1}, I_{2}\right)$ over a refined neutrosophic field $K\left(I_{1}, I_{2}\right)$ then

1. $U\left(I_{1}, I_{2}\right)$ is a strong refined neutrosophic subspace of $V\left(I_{1}, I_{2}\right)$ containing $v_{1}, v_{2}, \cdots, v_{n}$.

2. $U\left(I_{1}, I_{2}\right)$ is the smallest subspace containing $v 1, v_{2}, \cdots, v_{n}$ in the sense that any strong refined neutrosophic subspace of $V\left(I_{1}, I_{2}\right)$ that contains each of these refined neutrosophic vectors, must contain $U\left(I_{1}, I_{2}\right)$.

Proof. 1. (a) $U\left(I_{1}, I_{2}\right) \neq \emptyset$, since we can find $0=0+0 I_{1}+0 I_{2} \in K\left(I_{1}, I_{2}\right)$ such that $0=0 v_{1}+\cdots+0 v_{n}$ belongs to $U\left(I_{1}, I_{2}\right)$.

(b) Let $v, u \in U\left(I_{1}, I_{2}\right)$ where $u=s_{1} v_{1}+s_{2} v_{2}+\cdots+s_{n} v_{n}$ and $v=t_{1} v_{1}+t_{2} v_{2}+\cdots+t_{n} v_{n}$ and $\alpha=p+p_{1} I_{1}+p_{2} I_{2} \in K\left(I_{1}, I_{2}\right)$ then

$$
\begin{gathered}
u+v=\left(s_{1}+t_{1}\right) v_{1}+\left(s_{2}+t_{2}\right) v_{2}+\cdots+\left(s_{n}+t_{n}\right) v_{n}, \\
\alpha u=\left(\alpha s_{1}\right) v_{1}+\left(\alpha s_{2}\right) v_{2}+\cdots+\left(\alpha s_{n}\right) v_{n} .
\end{gathered}
$$

So both $u+v$ and $\alpha u$ lie in $U\left(I_{1}, I_{2}\right)$.

Finally, since $U \subseteq U(I)$, where $U$ is a vector space we conclude that $U\left(I_{1}, I_{2}\right)$ is a refined neutrosophic subspace.

2. Let $W\left(I_{1}, I_{2}\right)$ be a refined neutrosophic subspace of $V\left(I_{1}, I_{2}\right)$ that contains each of $v_{1}, v_{2}, \cdots, v_{n}$. Since $W\left(I_{1}, I_{2}\right)$ is closed under scalar multiplication, each of $\alpha_{1} v_{1}, \alpha_{2} v_{2}, \cdots, \alpha_{n} v_{n}$ lies in $W\left(I_{1}, I_{2}\right)$ for any choice of

$$
\alpha_{1}=p_{1}+q_{1} I_{1}+r_{1} I_{2}, \alpha_{2}=p_{2}+q_{2} I_{1}+r_{2} I_{2}, \cdots, \alpha_{n}=p_{n}+q_{n} I_{1}+r_{n} I_{2} \in K\left(I_{1}, I_{2}\right) .
$$

But then $\alpha v_{1}+\alpha_{2} v_{2}+\cdots+\alpha_{n} v_{n}$ lies in $W\left(I_{1}, I_{2}\right)$ since $W\left(I_{1}, I_{2}\right)$ is closed under addition. This means that $W\left(I_{1}, I_{2}\right)$ contains every member of $U\left(I_{1}, I_{2}\right)$, which proves (2).

Example 2.14. Let $P_{n}\left(I_{1}, I_{2}\right)$ be a strong refined neutrosophic vector space over a refined neutrosophic field $K\left(I_{1}, I_{2}\right)$. Then $P_{n}\left(I_{1}, I_{2}\right)=\operatorname{span}\left\{1, x, x^{2}, \cdots, x^{n}\right\}$.

We need only show that each neutrosophic polynomial $p(x)$ in $P_{n}\left(I_{1}, I_{2}\right)$ is a linear combination of $1, x, \cdots, x^{n}$. But this is clear because $p(x)$ has the form $p(x)=a_{0}+a_{1} x+a_{2} x^{2}+\cdots+a_{n} x^{2}$. With $a_{0}, a_{1}, \cdots, a_{n} \in K\left(I_{1}, I_{2}\right)$.

Example 2.15. Let $\mathbb{R}^{3}\left(I_{1}, I_{2}\right)$ be a strong refined neutrosophic vector space over a refined neutrosophic field $K\left(I_{1}, I_{2}\right)$. Then $\mathbb{R}^{3}\left(I_{1}, I_{2}\right)=\operatorname{span}\left\{\left(1+I_{1}+I_{2}\right),\left(1+I_{1}+0 I_{2}\right),\left(0+I_{1}+I_{2}\right)\right\}$.

Write $v_{1}=\left(1+I_{1}+I_{2}\right), v_{2}=\left(1+I_{1}+0 I_{2}\right), v_{3}=\left(0+I_{1}+I_{2}\right)$, and $U\left(I_{1}, I_{2}\right)=\operatorname{span}\left\{v_{1}, v_{2}, v_{3}\right\}$. Obviously $U\left(I_{1}, I_{2}\right)$ is contained in $\mathbb{R}^{3}\left(I_{1}, I_{2}\right)$.

We have $\mathbb{R}^{3}\left(I_{1}, I_{2}\right)=\operatorname{span}\left\{\left(1+0 I_{1}+0 I_{2}\right),\left(0+I_{1}+0 I_{2}\right),\left(0+0 I_{1}+I_{2}\right)\right\}$.

So to prove that $\mathbb{R}^{3}\left(I_{1}, I_{2}\right)$ is contained in $U\left(I_{1}, I_{2}\right)$, it is enough by Proposition 2.13 to show that each of 
$\left(1+0 I_{1}+0 I_{2}\right),\left(0+I_{1}+0 I_{2}\right),\left(0+0 I_{1}+I_{2}\right)$ lies in $\operatorname{span}\left\{v_{1}, v_{2}, v_{3}\right\}$. But they can be given explicitly as linear combinations of $v_{1}, v_{2}$, and $v_{3}$ :

$$
\begin{aligned}
& \left(1+0 I_{1}+0 I_{2}\right)=\left(1+I_{1}+I_{2}\right)-\left(0+I_{1}+I_{2}\right)=v_{1}-v_{3}, \\
& \left(0+0 I_{1}+I_{2}\right)=\left(1+I_{1}+I_{2}\right)-\left(1+I_{1}+0 I_{2}\right)=v_{1}-v_{2}
\end{aligned}
$$

and then, using the first of these, we have

$$
\left(0+I_{1}+0 I_{2}\right)=\left(1+I_{1}+0 I_{2}\right)-\left(1+0 I_{1}+0 I_{2}\right)=v_{2}-\left(v_{1}-v_{3}\right)=v_{2}-v_{2}+v_{3} .
$$

Proposition 2.16. Let $x=a+b I_{1}+c I_{2}$ and $y=d+e I_{1}+f I_{2}$ be two refined neutrosophic vectors in a strong refined neutrosophic vector space $V\left(I_{1}, I_{2}\right)$ over refined neutrosophic field $K\left(I_{1}, I_{2}\right)$.

Then span $\{x, y\}=\operatorname{span}\{x+y, x-y\}$, i.e.,

$\operatorname{span}\left\{a+b I_{1}+c I_{2}, d+e I_{1}+f I_{2}\right\}=\operatorname{span}\left\{a+d+(b+e) I_{1}+(c+f) I_{2}, a-d+(b-e) I_{1}+(c-f) I_{2}\right\}$.

Proof. We have

$\operatorname{span}\left\{a+d+(b+e) I_{1}+(c+f) I_{2}, a-d+(b-e) I_{1}+(c-f) I_{2}\right\} \subseteq \operatorname{span}\left\{a+b I_{1}+c I_{2}, d+e I_{1}+f I_{2}\right\}$ because both $a+d+(b+e) I_{1}+(c+f) I_{2}$ and $a-d+(b-e) I_{1}+(c-f) I_{2}$ lie in $\operatorname{span}\left\{a+b I_{1}+c I_{2}, d+e I_{1}+f I_{2}\right\}$. On the other hand,

$$
\begin{aligned}
a+b I_{1}+c I_{2} & =\frac{1}{2}\left[a+d+(b+e) I_{1}+(c+f) I_{2}\right]+\frac{1}{2}\left[a-d+(b-e) I_{1}+(c-f) I_{2}\right] \\
d+e I_{1}+f I_{2} & =\frac{1}{2}\left[a+d+(b+e) I_{1}+(c+f) I_{2}\right]-\frac{1}{2}\left[a-d+(b-e) I_{1}+(c-f) I_{2}\right],
\end{aligned}
$$

so

$\operatorname{span}\left\{a+b I_{1}+c I_{2}, d+e I_{1}+f I_{2}\right\} \subseteq \operatorname{span}\left\{a+d+(b+e) I_{1}+(c+f) I_{2}, a-d+(b-e) I_{1}+(c-f) I_{2}\right\}$ by Proposition 2.13 . Hence the prove.

Proposition 2.17. Let $U\left(I_{1}, I_{2}\right)$ and $W\left(I_{1}, I_{2}\right)$ be strong refined neutrosophic subspaces of as strong refined neutrosophic vector space $V\left(I_{1}, I_{2}\right)$ over a refined neutrosophic field $K\left(I_{1}, I_{2}\right)$. Then

1. $U\left(I_{1}, I_{2}\right) \subseteq W\left(I_{1}, I_{2}\right) \Longrightarrow \operatorname{span}\left(U\left(I_{1}, I_{2}\right)\right) \subseteq \operatorname{span}\left(W\left(I_{1}, I_{2}\right)\right)$.

2. $\operatorname{span}\left(\operatorname{span}\left(U\left(I_{1}, I_{2}\right)\right)\right)=\operatorname{span}\left(U\left(I_{1}, I_{2}\right)\right)$.

3. $\operatorname{span}\left(U\left(I_{1}, I_{2}\right) \cup W\left(I_{1}, I_{2}\right)\right)=\operatorname{span}\left(U\left(I_{1}, I_{2}\right)\right)+\operatorname{span}\left(W\left(I_{1}, I_{2}\right)\right)$.

Proof. The proof of 1, 2 and 3 are the same as in classical case.

Definition 2.18. Let $V\left(I_{1}, I_{2}\right)$ be a strong refined neutrosophic vector space over a refined neutrosophic field $K\left(I_{1}, I_{2}\right)$. A linearly independent subset $\mathbb{B}\left[I_{1}, I_{2}\right]=\left\{v_{1}, v_{2}, \cdots, v_{n}\right\}$ of $V\left(I_{1}, I_{2}\right)$ is called a basis for $V\left(I_{1}, I_{2}\right)$ if $B\left[I_{1}, I_{2}\right]$ spans $V\left(I_{1}, I_{2}\right)$.

Proposition 2.19. Let $V\left(I_{1}, I_{2}\right)$ be a strong refined neutrosophic vector space over a refined neutrosophic field $K\left(I_{1}, I_{2}\right)$. The bases of $V\left(I_{1}, I_{2}\right)$ are the same as the bases of $V$ over a field $K$.

Proof:

Suppose that $\mathbb{B}=\left\{v_{1}, v_{2}, \cdots, v_{n}\right\}$ is an arbitrary basis for $V$ over the field $K$. Let $v=a+b I_{1}+c I_{2}$ be an arbitrary element of $V\left(I_{1}, I_{2}\right)$ and let $\alpha_{1}=k_{1}+m_{1} I_{1}+t_{1} I_{2}, \alpha_{2}=k_{2}+m_{2} I_{1}+t_{2} I_{2} \cdots, \alpha_{n}=k_{n}+m_{n} I_{1}+t_{n} I_{2}$ be elements of $K\left(I_{1}, I_{2}\right)$. Then from $\alpha_{1} v_{1}+\alpha_{2} v_{2}+\cdots+\alpha_{n} v_{n}=0$, we obtain

$$
\begin{gathered}
k_{1} v_{1}+k_{2} v_{2}+\cdots+k_{n} v_{n}=0, \\
m_{1} v_{1}+m_{2} v_{2}+\cdots+m_{n} v_{n}=0 \\
t_{1} v_{1}+t_{2} v_{2}+\cdots+t_{n} v_{n}=0
\end{gathered}
$$

Since $v_{i} s$ are linearly independent, we have $k_{i}=0, m_{j}=0$ and $t_{z}=0 \quad$ where $i, j, z=1,2, \cdots, n$. Hence, $\alpha_{i}=0, i=1,2, \cdots, n$. This shows that $\mathbb{B}$ is also a linearly independent set in $V\left(I_{1}, I_{2}\right)$.

To show that B spans $V\left(I_{1}, I_{2}\right)$, let $v=a+b I_{1}+c I_{2}=\alpha_{1} v_{1}+\alpha_{2} v_{2}+\cdots+\alpha_{n} v_{n}$.

Then we have

$$
a=k_{1} v_{1}+k_{2} v_{2}+\cdots+k_{n} v_{n}
$$




$$
\begin{aligned}
& b=m_{1} v_{1}+m_{2} v_{2}+\cdots+m_{n} v_{n}, \\
& c=t_{1} v_{1}+t_{2} v_{2}+\cdots+t_{n} v_{n}=0 .
\end{aligned}
$$

Since $a, b, c \in V$, it follows that $v=a+b I_{1}+c I_{2}$ can be written uniquely as a linear combination of $v_{i} s$. Hence, $\mathbb{B}$ is a basis for $V\left(I_{1}, I_{2}\right)$. Since $\mathbb{B}$ is arbitrary, the required result follows;

Proposition 2.20. Let $V\left(I_{1}, I_{2}\right)$ be a strong refined neutrosophic vector space over a refined neutrosophic field $K\left(I_{1}, I_{2}\right)$ which is spanned by a finite set of neutrosophic vectors $v_{1}, v_{2}, \cdots, v_{m}$. Then any independent set of refined neutrosophic vectors in $V\left(I_{1}, I_{2}\right)$ is finite and contains no more than $m$ elements.

Proof: Let $v=a+b I_{1}+c I_{2}, u=d+e I_{1}+f I_{2}$.

To prove this it suffices to show that every refined neutrosophic subset $S\left(I_{1}, I_{2}\right)$ of $V\left(I_{1}, I_{2}\right)$ which contains more than $m$ refined neutrosophic vectors is linearly dependent.

Let $S\left(I_{1}, I_{2}\right)$ be such a set. In $S\left(I_{1}, I_{2}\right)$ there are distinct refined neutrosophic vectors $u_{1}, u_{2}, \cdots, u_{n}$ where $n>m$.

Since $v_{1}, v_{2}, \cdots, v_{m}$ span $V\left(I_{1}, I_{2}\right)$, there exist scalars $C_{i j}$ with $C=r+s I_{1}+t I_{2} \in K\left(I_{1}, I_{2}\right)$ such that

$$
u_{j}=\sum_{i=1}^{m} C_{i j} v_{i}=\sum_{i=1}^{m}\left(r_{i j} a_{i}+\left(r_{i j} b_{i}+s_{i j} a_{i}+s_{i j} b_{i}+s_{i j} c_{i}+t_{i j} b_{i}\right) I_{1}+\left(r_{i j} c_{i}+t_{i j} a_{i}+t_{i j} c_{i}\right) I_{2}\right) .
$$

For any n scalars $x_{1}, x_{2}, \cdots, x_{n}$ with $x=p+q I_{1}+z I_{2} \in K\left(I_{1}, I_{2}\right)$ we have

$$
\begin{aligned}
x_{1} u_{1} & +x_{2} u_{2}+\cdots+x_{n} u_{n}=\sum_{j=1}^{n} x_{j} u_{j} \\
= & \sum_{j=1}^{n}\left(p_{j}+q_{j} I_{1}+z_{j} I_{2}\right) u_{j} \\
= & \sum_{j=1}^{n}\left(p_{j}+q_{j} I_{1}+z_{j} I_{2}\right) \sum_{i=1}^{m} C_{i j} v_{i} \\
= & \sum_{j=1}^{n}\left(p_{j}+q_{j} I_{1}+z_{j} I_{2}\right) \sum_{i=1}^{m}\left(r_{i j} a_{i}+\left(r_{i j} b_{i}+s_{i j} a_{i}+s_{i j} b_{i}+s_{i j} c_{i}+\right.\right. \\
= & \left.\left.t_{i j} b_{i}\right) I_{1}+\left(r_{i j} c_{i}+t_{i j} a_{i}+t_{i j} c_{i}\right) I_{2}\right) \\
= & \sum_{j=1}^{n} \sum_{i=1}^{m}\left(p_{j}+q_{j} I_{1}+z_{j} I_{2}\right)\left(r_{i j} a_{i}+\left(r_{i j} b_{i}+s_{i j} a_{i}+s_{i j} b_{i}+s_{i j} c_{i}+t_{i j} b_{i}\right) I_{1}+\right. \\
= & \left.\left.\sum_{j=1}^{n} c_{i=1}+t_{i j} a_{i}+t_{i j} c_{i}\right) I_{2}\right) \\
& q_{j} s_{i j} a_{i}+q_{j} s_{i j} b_{i}+q_{j}+\left(p_{j} r_{i j} b_{i}+p_{j} s_{i j} a_{i}+p_{j} s_{i j} b_{i}+p_{j} s_{i j} s_{i j} c_{i}+p_{j} t_{i j} b_{i}+q_{i} c_{i} r_{i j} a_{i}+q_{j} t_{i j} a_{i}+q_{j} t_{i j} b_{i}+z_{j} r_{i j} b_{i}+z_{j} s_{i j} a_{i}+z_{j} s_{i j} b_{i}\right. \\
& \left.\left.+z_{j} s_{i j} c_{i}+z_{j} t_{i j} b_{i}\right) I_{1}+\left(p_{j} r_{i j} c_{i}+p_{j} t_{i j} a_{i}+p_{j} t_{i j} c_{i}+z_{j} r_{i j} a_{i}+z_{j} r_{i j} c_{i}+z_{j} t_{i j} a_{i}+z_{j} t_{i j} c_{i}\right) I_{2}\right) \\
= & \sum_{j=1}^{n} \sum_{i=1}^{m}\left(\left(p_{j}+q_{j} I_{1}+z_{j} I_{2}\right)\left(r_{i j}+s_{i j} I_{1}+t_{i j} I_{2}\right)\left(a_{i}+b I_{1}+b I_{2}\right)\right) \\
= & \sum_{j=1}^{n} \sum_{i=1}^{m}\left(\left(p_{j}+q_{j} I_{1}+z_{j} I_{2}\right)\left(r_{i j}+s_{i j} I_{1}+t_{i j} I_{2}\right)\right)\left(a_{i}+b I_{1}+b I_{2}\right) \\
= & \sum_{j=1}^{n} \sum_{i=1}^{m}\left(C_{i j} x_{j}\right) v_{i} \\
= & \sum_{i=1}^{m}\left(\sum_{j=1}^{n} C_{i j} x_{j}\right) v_{i} .
\end{aligned}
$$

Since $n>m$, there exist scalars $x_{1}, x_{2}, \cdots, x_{n}$ not all 0 such that

$$
\sum_{j=1}^{n} C_{i j} x_{j}=0 \quad 1 \leq i \leq m
$$

Hence $x_{1} u_{1}+x_{2} u_{2}+\cdots+x_{n} u_{n}=0$. This shows that $S\left(I_{1}, I_{2}\right)$ is a linearly dependent set.

Definition 2.21. Let $V\left(I_{1}, I_{2}\right)$ be a strong refined neutrosophic vector space over a neutrosophic field $K\left(I_{1}, I_{2}\right)$. The number of elements in the basis for $V\left(I_{1}, I_{2}\right)$ is called the dimension of $V\left(I_{1}, I_{2}\right)$ and it is denoted by $\operatorname{dim}_{s}\left(V\left(I_{1}, I_{2}\right)\right)$. If the number of elements in the basis for $V\left(I_{1}, I_{2}\right)$ is finite, $V\left(I_{1}, I_{2}\right)$ is called a finite dimensional strong refined neutrosophic vector space. Otherwise, $V\left(I_{1}, I_{2}\right)$ is called an infinite dimensional strong refined neutrosophic vector space.

Definition 2.22. Let $V\left(I_{1}, I_{2}\right)$ be a weak refined neutrosophic vector space over a field $K$. The number of elements in the basis for $V\left(I_{1}, I_{2}\right)$ is called the dimension of $V\left(I_{1}, I_{2}\right)$ and it is denoted by $\operatorname{dim}_{w}\left(V\left(I_{1}, I_{2}\right)\right)$. If the number of elements in the basis for $V\left(I_{1}, I_{2}\right)$ is finite, $V\left(I_{1}, I_{2}\right)$ is called a finite dimensional weak refined neutrosophic vector space. Otherwise, $V\left(I_{1}, I_{2}\right)$ is called an infinite dimensional weak refined neutrosophic vector space.

Example 2.23. The strong refined neutrosophic vector space of Example 2.14 is finite dimensional and $\operatorname{dim}_{s}\left(V\left(I_{1}, I_{2}\right)\right)=n+1$.

Proposition 2.24. Let $V\left(I_{1}, I_{2}\right)$ be a finite dimensional strong refined neutrosophic vector space over a refined neutrosophic field $K\left(I_{1}, I_{2}\right)$. Then every basis of $V\left(I_{1}, I_{2}\right)$ has the same number of elements.

Proof. The proof is similar to the proof in classical case. 
Proposition 2.25. Let $V\left(I_{1}, I_{2}\right)$ be a finite dimensional weak (strong) refined neutrosophic vector space over a field $K$ (resp. over a refined neutrosophic field $K\left(I_{1}, I_{2}\right)$ ). If $\operatorname{dim}_{s}\left(V\left(I_{1}, I_{2}\right)\right)=n$, then $\operatorname{dim}_{w}\left(V\left(I_{1}, I_{2}\right)\right)=2 n$.

This can be easily seen in the examples given below.

Example 2.26. Let $V\left(I_{1}, 1_{2}\right)=\mathbb{R}^{n}\left(I_{1}, I_{2}\right)$ be a strong refined neutrosophic vector space over a refined neutrosophic field $R\left(I_{1}, 1_{2}\right)$.

The set $\mathbb{B}=\left\{v_{1}=(1,0,0, \cdots, 0), v_{2}=(0,1,0, \cdots, 0), \cdots, v_{n}=(0,0,0, \cdots, 1)\right\}$ is a basis for $V\left(I_{1}, I_{2}\right)$.

Example 2.27. Let $V\left(I_{1}, I_{2}\right)=\mathbb{R}^{n}\left(I_{1}, I_{2}\right)$ be a weak refined neutrosophic vector space over $\mathbb{R}$. The set $\mathbb{B}=\left\{v_{1}=(1,0,0, \cdots, 0), v_{2}=(0,1,0, \cdots, 0), \cdots, v_{k}=(0,0,0, \cdots, 1)\right.$,

$\left.v_{k+1}=\left(I_{1}+I_{2}, 0,0, \cdots, 0\right), v_{k+2}=\left(0, I_{1}+I_{2}, 0, \cdots, 0\right), \cdots, v_{n}=\left(0,0,0, \cdots, I_{1}+I_{2}\right)\right\}$ is a basis for $V\left(I_{1}, I_{2}\right)$.

Note 2. The bases of the strong refined neutrosophic vector space of Example 2.26 is contained in the bases of the weak refined neutrosophic vector space of Example 2.27. This observation is recorded in the next proposition.

Proposition 2.28. Let $V\left(I_{1}, I_{2}\right)$ be a strong refined neutrosophic vector space over a refined neutrosophic field $K\left(I_{1}, I_{2}\right)$. Then the bases of $V\left(I_{1}, I_{2}\right)$ over $K\left(I_{1}, I_{2}\right)$ are contained in the bases of the weak refined neutrosophic vector space $V\left(I_{1}, I_{2}\right)$ over a field $K$.

Proof. The proof follows from Examples 2.26 and 2.27 .

Proposition 2.29. Let $W\left(I_{1}, I_{2}\right)$ be a strong refined neutrosophic subspace of a finite dimensional strong refined neutrosophic vector space $V\left(I_{1}, I_{2}\right)$ over a neutrosophic field $K\left(I_{1}, I_{2}\right)$. Then $W\left(I_{1}, I_{2}\right)$ is finite dimensional and $\operatorname{dim}_{s}\left(W\left(I_{1}, I_{2}\right)\right) \leq \operatorname{dim}_{s}\left(V\left(I_{1}, I_{2}\right)\right)$. If $\operatorname{dim}_{s}\left(W\left(I_{1}, I_{2}\right)\right)=\operatorname{dim}_{s}\left(V\left(I_{1}, I_{2}\right)\right)$, then

$W\left(I_{1}, I_{2}\right)=V\left(I_{1}, I_{2}\right)$.

Proof. If $W\left(I_{1}, I_{2}\right)=\{\}, \operatorname{dim}_{s} W\left(I_{1}, I_{2}\right)=0$. So assume $W\left(I_{1}, I_{2}\right) \neq\{\}$, and choose $u_{1} \neq 0$ in $W\left(I_{1}, I_{2}\right)$. If $W\left(I_{1}, I_{2}\right)=\operatorname{span}\left\{u_{1}\right\}$, then $\operatorname{dim}_{s} W\left(I_{1}, I_{2}\right)=1$. If $W\left(I_{1}, I_{2}\right) \neq \operatorname{span}\left\{u_{1}\right\}$, choose $u_{2}$ in $W\left(I_{1}, I_{2}\right)$ outside $\operatorname{span}\left\{u_{1}\right\}$. Then $\left\{u_{1}, u_{2}\right\}$, is linearly independent.

If $W\left(I_{1}, I_{2}\right)=$ span $\left\{u_{1}, u_{2}\right\}$, then $\operatorname{dim}_{s} W\left(I_{1}, I_{2}\right)=2$. If not, repeat the process to find $u_{3}$ in $W\left(I_{1}, I_{2}\right)$ such that $\left\{u_{1}, u_{2}, u_{3}\right\}$ is linearly independent. Continue in this way. The process must terminate because the refined neutrosophic space $V\left(I_{1}, I_{2}\right)$ (having dimension $\mathrm{n}$ ) cannot contain more than $n$ independent vectors. Hence $W\left(I_{1}, I_{2}\right)$ has a basis of at most $n$ refined neutrosophic vectors.

Secondly, Let $\operatorname{dim}_{s} W\left(I_{1}, I_{2}\right)=\operatorname{dim}_{s} V\left(I_{1}, I_{2}\right)=m$. Then any basis $\left\{u_{1}, \cdots, u_{m}\right\}$ of $W\left(I_{1}, I_{2}\right)$ is an independent set of $\mathrm{m}$ refined neutrosophic vectors in $V\left(I_{1}, I_{2}\right)$ and so is a basis of $V\left(I_{1}, I_{2}\right)$.

In particular, $\left\{u_{1}, \cdots, u_{m}\right\}$ spans $V\left(I_{1}, I_{2}\right)$ so, because it also spans $W\left(I_{1}, I_{2}\right)$,

$V\left(I_{1}, I_{2}\right)=\operatorname{span}\left\{u_{1}, \cdots, u_{m}\right\}=W\left(I_{1}, I_{2}\right)$.

Proposition 2.30. Let $U\left(I_{1}, I_{2}\right)$ and $W\left(I_{1}, I_{2}\right)$ be finite dimensional strong refined neutrosophic subspaces of a strong refined neutrosophic vector space $V\left(I_{1}, I_{2}\right)$ over a refined neutrosophic field $K\left(I_{1}, I_{2}\right)$. Then $U\left(I_{1}, I_{2}\right)+W\left(I_{1}, I_{2}\right)$ is a finite dimensional strong refined neutrosophic subspace of $V\left(I_{1}, I_{2}\right)$ and

$$
\begin{aligned}
& \quad \operatorname{dim}_{s}\left(U\left(I_{1}, I_{2}\right)+W\left(I_{1}, I_{2}\right)\right)=\operatorname{dim}_{s}\left(U\left(I_{1}, I_{2}\right)\right)+\operatorname{dim}_{s}\left(W\left(I_{1}, I_{2}\right)\right)-\operatorname{dim}_{s}\left(U\left(I_{1}, I_{2}\right) \cap W\left(I_{1}, I_{2}\right)\right) . \\
& \text { If } V\left(I_{1}, I_{2}\right)=U\left(I_{1}, I_{2}\right) \oplus W\left(I_{1}, I_{2}\right) \text { then } \\
& \qquad \operatorname{dim}_{s}\left(U\left(I_{1}, I_{2}\right)+W\left(I_{1}, I_{2}\right)\right)=\operatorname{dim}_{s}\left(U\left(I_{1}, I_{2}\right)\right)+\operatorname{dim}_{s}\left(W\left(I_{1}, I_{2}\right)\right) .
\end{aligned}
$$

Definition 2.31. Let $V\left(I_{1}, I_{2}\right)$ and $W\left(I_{1}, I_{2}\right)$ be strong refined neutrosophic vector spaces over a refined neutrosophic field $K\left(I_{1}, I_{2}\right)$ and let $\phi: V\left(I_{1}, I_{2}\right) \longrightarrow W\left(I_{1}, I_{2}\right)$ be a mapping of $V\left(I_{1}, I_{2}\right)$ into $W\left(I_{1}, I_{2}\right)$. $\phi$ is called a refined neutrosophic vector space homomorphism if the following conditions hold:

1. $\phi$ is a vector space homomorphism.

2. $\phi\left(I_{k}\right)=I_{k}$ for $k=1,2$.

If $\phi$ is a bijective refined neutrosophic vector space homomorphism, then $\phi$ is called a refined neutrosophic vector space isomorphism and we write $V\left(I_{1}, I_{2}\right) \cong W\left(I_{1}, I_{2}\right)$. 
Definition 2.32. Let $V\left(I_{1}, I_{2}\right)$ and $W\left(I_{1}, I_{2}\right)$ be strong refined neutrosophic vector spaces over a refined neutrosophic field $K\left(I_{1}, I_{2}\right)$ and let $\phi: V\left(I_{1}, I_{2}\right) \longrightarrow W\left(I_{1}, I_{2}\right)$ be a refined neutrosophic vector space homomorphism.

1. The kernel of $\phi$ denoted by $\operatorname{Ker} \phi$ is defined by the set $\left\{v \in V\left(I_{1}, I_{2}\right): \phi(v)=0\right\}$.

2. The image of $\phi$ denoted by $\operatorname{Im} \phi$ is defined by the set $\left\{w \in W\left(I_{1}, I_{2}\right): \phi(v)=w\right.$ for some $\left.\left.v \in V\left(I_{1}, I_{2}\right)\right)\right\}$.

Example 2.33. Let $V\left(I_{1}, I_{2}\right)$ be a strong refined neutrosophic vector space over a refined neutrosophic field $K\left(I_{1}, I_{2}\right)$

1. The mapping $\phi: V\left(I_{1}, I_{1}\right) \longrightarrow V\left(I_{1}, I_{1}\right)$ defined by $\phi(v)=v$ for all $v=a+b I_{1}+c I_{2} \in V\left(I_{1}, I_{2}\right)$ is a refined neutrosophic vector space homomorphism and $\operatorname{Ker} \phi=\{0\}$.

2. The mapping $\phi: V\left(I_{1}, I_{1}\right) \longrightarrow V\left(I_{1}, I_{1}\right)$ defined by $\phi(v)=0$ for all $v=a+b I_{1}+c I_{2} \in V\left(I_{1}, I_{2}\right)$ is not a refined neutrosophic vector space homomorphism. Since for $I_{k} \in V\left(I_{1}, I_{2}\right), \phi\left(I_{k}\right) \neq 0$.

Proposition 2.34. Let $V\left(I_{1}, I_{2}\right)$ and $W\left(I_{1}, I_{2}\right)$ be strong refined neutrosophic vector spaces over a neutrosophic field $K\left(I_{1}, I_{2}\right)$ and let $\phi: V\left(I_{1}, I_{2}\right) \longrightarrow W\left(I_{1}, I_{2}\right)$ be a refined neutrosophic vector space homomorphism. Then

1. Ker $\phi$ is not a strong refined neutrosophic subspace of $V\left(I_{1}, I_{2}\right)$ but a subspace of $V\left(I_{1}, I_{2}\right)$.

2. Im $\phi$ is a strong refined neutrosophic subspace of $W\left(I_{1}, I_{2}\right)$.

Proof. That $\operatorname{Ker} \phi$ is a subspace of $V\left(I_{1}, I_{2}\right)$, and $\operatorname{Im} \phi$ is a strong refined neutrosophic subspace of $W\left(I_{1}, I_{2}\right)$ follows easily .

Now, to show that $\operatorname{Ker} \phi$ is not a strong refined neutrosophic subspace of $V\left(I_{1}, I_{2}\right)$, we note that for $I_{k} \in V\left(I_{1}, I_{2}\right)$ we have that $\phi\left(I_{k}\right)=I_{k} \neq 0$, this implies that $I_{k} \notin k e r \phi$.

Hence, $\operatorname{ker} \phi$ is not a strong refined neutrosophic subspace.

Proposition 2.35. Let $V\left(I_{1}, I_{2}\right)$ and $W\left(I_{1}, I_{2}\right)$ be strong refined neutrosophic vector spaces over a refined neutrosophic field $K\left(I_{1}, I_{2}\right)$ and let $\phi: V\left(I_{1}, I_{2}\right) \longrightarrow W\left(I_{1}, I_{2}\right)$ be a refined neutrosophic vector space homomorphism. If $\mathbb{B}=\left\{v_{1}, v_{2}, \cdots, v_{n}\right\}$ is a basis for $V\left(I_{1}, I_{2}\right)$, then $\phi(\mathbb{B})=\left\{\phi\left(v_{1}\right), \phi\left(v_{2}\right), \cdots, \phi\left(v_{n}\right)\right\}$ is a basis for $W\left(I_{1}, I_{2}\right)$.

Proof. Since $\mathbb{B}=\left\{v_{1}, v_{2}, \cdots, v_{n}\right\}$ is a basis for $V\left(I_{1}, I_{2}\right)$, it spans $V\left(I_{1}, I_{2}\right)$, so for every

$v \in V\left(I_{1}, I_{2}\right)$, there exist $\alpha_{i} \in K\left(I_{1}, I_{2}\right)$, with $i=1,2,3, \cdots n$ such that $v=\alpha_{1} v_{1}+\alpha_{2} v_{2}+\cdots+\alpha_{n} v_{n}$.

$\phi(v)=\phi\left(\alpha_{1} v_{1}+\alpha_{2} v_{2}+\cdots+\alpha_{n} v_{n}\right)$

$=\phi\left(\alpha_{1} v_{1}\right)+\phi\left(\alpha_{2} v_{2}\right)+\cdots+\phi\left(\alpha_{n} v_{n}\right)$

$=\alpha_{1} \phi\left(v_{1}\right)+\alpha_{2} \phi\left(v_{2}\right)+\cdots+\alpha_{n} \phi\left(v_{n}\right)$.

Thus $\phi(v)=\alpha_{1} \phi\left(v_{1}\right)+\alpha_{2} \phi\left(v_{2}\right)+\cdots+\alpha_{n} \phi\left(v_{n}\right)$.

Then for every $v \in V\left(I_{1}, I_{2}\right)$, its image $\phi(v) \in W\left(I_{1}, I_{2}\right)$ can be written as a linear combination of $\left\{\phi\left(v_{1}\right), \phi\left(v_{2}\right), \cdots \phi\left(v_{n}\right)\right\}$. Hence $\left\{\phi\left(v_{1}\right), \phi\left(v_{2}\right), \cdots \phi\left(v_{n}\right)\right\}$ spans $W\left(I_{1}, I_{2}\right)$.

Now if $\alpha_{1} \phi\left(v_{1}\right)+\alpha_{2} \phi\left(v_{2}\right)+\cdots+\alpha_{n} \phi\left(v_{n}\right)=0$ then $\phi\left(\alpha_{1} v_{1}+\alpha_{2} v_{2}+\cdots+\alpha_{n} v_{n}\right)=0$.

But then each $\alpha_{i}=0$ by the independence of the $v_{i}$ so $\left\{\phi\left(v_{1}\right), \phi\left(v_{2}\right), \cdots \phi\left(v_{n}\right)\right\}$ is linearly independent.

To this end we can conclude that $\phi(\mathbb{B})=\left\{\phi\left(v_{1}\right), \phi\left(v_{2}\right), \cdots \phi\left(v_{n}\right)\right\}$ is a basis for $W\left(I_{1}, I_{2}\right)$.

Proposition 2.36. Let $W\left(I_{1}, I_{2}\right)$ be a strong refined neutrosophic subspace of a strong refined neutrosophic vector space $V\left(I_{1}, I_{2}\right)$ over a neutrosophic field $K\left(I_{1}, I_{2}\right)$. Let $\phi: V\left(I_{1}, I_{2}\right) \longrightarrow V\left(I_{1}, I_{2}\right) / W\left(I_{1}, I_{2}\right)$ be a mapping defined by $\phi(v)=v+W\left(I_{1}, I_{2}\right)$ for all $v \in V\left(I_{1}, I_{2}\right)$. Then $\phi$ is not a neutrosophic vector space homomorphism.

Proof. It is easily seen, since for $k=1,2, \phi\left(I_{k}\right)=I_{k}+W\left(I_{1}, I_{2}\right)=W\left(I_{1}, I_{2}\right) \neq I_{k}$.

Remark 2.37. One of the natural questions would be if $V\left(I_{1}, I_{2}\right)$ and $W\left(I_{1}, I_{2}\right)$ are strong (weak) refined neutrosophic vector spaces over a refined neutrosophic field $K\left(I_{1}, I_{2}\right)($ respectively $(K))$. Suppose $\operatorname{Hom}\left(V\left(I_{1}, I_{2}\right), W\left(I_{1}, I_{2}\right)\right)$ is the collection of all refined neutrosophic vector space homomorphisms from $V\left(I_{1}, I_{2}\right)$ into $W\left(I_{1}, I_{2}\right)$, then by defining + and scalar multiplication on $\operatorname{Hom}\left(V\left(I_{1}, I_{2}\right), W\left(I_{1}, I_{2}\right)\right)$ can we obtain a refined neutrosophic vector? The answer to this is given in the next proposition . 
Proposition 2.38. Let $V\left(I_{I}, I_{2}\right)$ and $W\left(I_{1}, I_{2}\right)$ be any two strong refined neutrosophic vector spaces over the refined neutrosophic field $K\left(I_{1}, I_{2}\right)$. Let $\operatorname{Hom}\left(V\left(I_{1}, I_{2}\right), W\left(I_{1}, I_{2}\right)\right)$ be the collection of all refined neutrosophic vector space homomorphisms from $V\left(I_{1}, I_{2}\right)$ into $W\left(I_{1}, I_{2}\right)$, then the triple

$\left(H o m\left(V\left(I_{1}, I_{2}\right), W\left(I_{1}, I_{2}\right)\right),+, \cdot\right)$ is not a refined neutrosophic vector space over $K\left(I_{1}, I_{2}\right)$.

Proof. $\phi, \psi \in \operatorname{Hom}\left(V\left(I_{1}, I_{2}\right), W\left(I_{1}, I_{2}\right)\right)$ then $(\phi+\psi)$ and $(\psi \phi) \in \operatorname{Hom}\left(V\left(I_{1}, I_{2}\right), W\left(I_{1}, I_{2}\right)\right)$, since $(\phi+\psi)\left(I_{k}\right)=\phi\left(I_{k}\right)+\psi\left(I_{k}\right)=I_{k}+I_{k}=2 I_{k} \neq I_{k}$ and $(\alpha \phi)\left(I_{k}\right)=\alpha \phi\left(I_{k}\right)=\alpha \phi\left(I_{k}\right) \neq I_{k}$ for all $\alpha \in K\left(I_{1}, I_{2}\right)$ and $k=1,2$.

Definition 2.39. Let $U\left(I_{1}, I_{2}\right), V\left(I_{1}, I_{2}\right)$ and $W\left(I_{1}, I_{2}\right)$ be strong refined neutrosophic vector spaces over a refined neutrosophic field $K\left(I_{1}, I_{2}\right)$ and let $\phi: U\left(I_{1}, I_{2}\right) \longrightarrow V\left(I_{1}, I_{2}\right), \psi: V\left(I_{1}, I_{2}\right) \longrightarrow W\left(I_{1}, I_{2}\right)$ be refined neutrosophic vector space homomorphisms.

The composition $\psi \phi: U\left(I_{1}, I_{2}\right) \longrightarrow W\left(I_{1}, I_{2}\right)$ is defined by $\psi \phi(u)=\psi(\phi(u))$ for all $u \in U\left(I_{1}, I_{2}\right)$.

Proposition 2.40. Let $U\left(I_{1}, I_{2}\right), V\left(I_{1}, I_{2}\right)$ and $W\left(I_{1}, I_{2}\right)$ be strong refined neutrosophic vector spaces over a refined neutrosophic field $K\left(I_{1}, I_{2}\right)$ and let $\phi: U\left(I_{1}, I_{2}\right) \longrightarrow V\left(I_{1}, I_{2}\right), \psi: V\left(I_{1}, I_{2}\right) \longrightarrow W\left(I_{1}, I_{2}\right)$ be refined neutrosophic vector space homomorphisms. Then the composition $\psi \phi: U\left(I_{1}, I_{2}\right) \longrightarrow W\left(I_{1}, I_{2}\right)$ is a refined neutrosophic vector space homomorphism.

Proof: That $\psi \phi$ is a vector space homomorphism is clear. Then for $u=I_{k} \in U\left(I_{1}, I_{2}\right)$, we have

$$
\psi \phi\left(I_{k}\right)=\psi\left(\phi\left(I_{k}\right)\right)=\phi\left(I_{k}\right)=I_{k} \text { with } k=1,2 .
$$

Hence $\psi \phi$ is a neutrosophic vector space homomorphism.

Let $V\left(I_{1}, I_{2}\right)$ be a strong refined neutrosophic vector space over a refined neutrosophic field $K\left(I_{1}, I_{2}\right)$ and let $\beta: V\left(I_{1}, I_{2}\right) \longrightarrow V\left(I_{1}, I_{2}\right)$ be a refined neutrosophic vector space homomorphism. If $\mathbb{B}=\left\{v_{1}, v_{2} \cdots, v_{n}\right\}$ is a basis for $V\left(I_{1}, I_{2}\right)$, then each $\beta\left(v_{i}\right) \in V\left(I_{1}, I_{2}\right)$ and thus for $\beta_{i j} \in K\left(I_{1}, I_{2}\right)$, we can write

$$
\begin{aligned}
& \beta\left(v_{1}\right)=\beta_{11} v_{1}+\beta_{12} v_{2}+\cdots+\beta_{1 n} v_{n} \\
& \beta\left(v_{2}\right)=\beta_{21} v_{1}+\beta_{22} v_{2}+\cdots+\beta_{2 n} v_{n}
\end{aligned}
$$

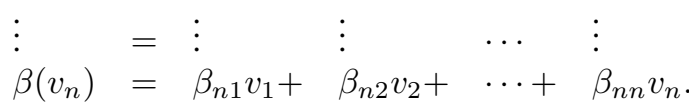

Let

$$
[\beta]_{\mathbb{B}}=\left[\begin{array}{llll}
\beta_{11} & \beta_{21} & \cdots & \beta_{n 1} \\
\beta_{12} & \beta_{22} & \cdots & \beta_{n 2} \\
\vdots & \vdots & \vdots & \vdots \\
\beta_{1 n} & \beta_{2 n} & \cdots & \beta_{n m}
\end{array}\right] .
$$

$[\beta]_{\mathbb{B}}$ is called the matrix representation of $\beta$ relative to the basis $\mathbb{B}$.

Proposition 2.41. Let $V\left(I_{1}, I_{2}\right)$ be a strong refined neutrosophic vector space over a refined neutrosophic field $K\left(I_{1}, I_{2}\right)$ and let $\beta: V\left(I_{1}, I_{2}\right) \longrightarrow V\left(I_{1}, I_{2}\right)$ be a refined neutrosophic vector space homomorphism. If $\mathbb{B}$ is a basis for $V\left(I_{1}, I_{2}\right)$ and $v$ is any element of $V\left(I_{1}, I_{2}\right)$, then

$$
[\beta]_{\mathbb{B}}[v]_{\mathbb{B}}=[\beta(v)]_{\mathbb{B}} .
$$

We give an example to help establish this proposition.

Example 2.42. Let $V\left(I_{1}, I_{2}\right)=\mathbb{R}^{3}\left(I_{1}, I_{2}\right)$ be a strong refined neutrosophic vector space over a refined neutrosophic field $K\left(I_{1}, I_{2}\right)=\mathbb{R}\left(I_{1}, I_{2}\right)$ and

let $v=\left(2+3 I_{1}+I_{2}, 4+3 I_{1}-I_{2}, 2+4 I_{1}+4 I_{2}\right) \in V\left(I_{1}, I_{2}\right)$. If $\beta: V\left(I_{1}, I_{2}\right) \longrightarrow V\left(I_{1}, I_{2}\right)$ is a refined neutrosophic vector space homomorphism defined by $\beta(v)=v$ for all $v \in V\left(I_{1}, I_{2}\right)$, then relative to the basis $\mathbb{B}=\left\{v_{1}=(1,1,0), v_{2}=(1,0,1), v_{3}=(0,1,1)\right\}$ for $V\left(I_{1}, I_{2}\right)$, the matrix of $\beta$ is obtained as

$$
[\beta]_{\mathbb{B}}=\left[\begin{array}{lll}
1+0 I_{1}+0 I_{2} & 1+0 I_{1}+0 I_{2} & 0+0 I_{1}+0 I_{2} \\
1+0 I_{1}+0 I_{2} & 0+0 I_{1}+0 I_{2} & 1+0 I_{1}+0 I_{2} \\
0+0 I_{1}+0 I_{2} & 1+0 I_{1}+0 I_{2} & 1+0 I_{1}+0 I_{2}
\end{array}\right] .
$$


For $v=\left(2+3 I_{1}+I_{2}, 4+3 I_{1}-I_{2}, 2+4 I_{1}+4 I_{2}\right) \in V\left(I_{1}, I_{2}\right)$, we have $\beta(v)=v=\left(2+I_{1}-2 I_{2}\right) v_{1}+\left(2 I_{1}+3 I_{2}\right) v_{2}+\left(2+2 I_{2}+I_{2}\right) v_{3}$

So that

$$
[v]_{\mathbb{B}}=\left[\begin{array}{l}
2+I_{1}-2 I_{2} \\
2 I_{1}+3 I_{2} \\
2+2 I_{1}+I_{2}
\end{array}\right]=[\beta(v)]_{\mathbb{B}}
$$

and we have

$$
[\beta]_{\mathbb{B}}[v]_{\mathbb{B}}=[\beta(v)]_{\mathbb{B}} .
$$

Example 2.43. Let $V\left(I_{1}, I_{2}\right)=\mathbb{R}^{2}\left(I_{1}, I_{2}\right)$ be a weak refined neutrosophic vector space over a field $K=R$ and let $v=\left(1-3 I_{1}+2 I_{2}, 3+I_{1}-4 I_{2}\right) \in V\left(I_{1}, I_{2}\right)$.

If $\beta: V\left(I_{1}, I_{2}\right) \longrightarrow V\left(I_{1}, I_{2}\right)$ is a refined neutrosophic vector space homomorphism defined by $\beta(v)=v$ for all $v \in V\left(I_{1}, I_{2}\right)$, then relative to the basis

$\mathbb{B}=\left\{v_{1}=(1,0), v_{2}=(0,1), v_{3}=\left(I_{1}, 0\right), v_{4}=\left(0, I_{1}\right), v_{5}=\left(I_{2}, 0\right), v_{6}=\left(0, I_{2}\right)\right\}$ for $V\left(I_{1}, I_{2}\right)$, the matrix of $\beta$ is obtained as

$$
[\beta]_{\mathbb{B}}=\left[\begin{array}{llllll}
1 & 0 & 0 & 0 & 0 & 0 \\
0 & 1 & 0 & 0 & 0 & 0 \\
0 & 0 & 1 & 0 & 0 & 0 \\
0 & 0 & 0 & 1 & 0 & 0 \\
0 & 0 & 0 & 0 & 1 & 0 \\
0 & 0 & 0 & 0 & 0 & 1
\end{array}\right] .
$$

For $v=\left(1-3 I_{1}+2 I_{2}, 3+I_{1}-4 I_{2}\right) \in V\left(I_{1}, I_{2}\right)$, we have

$$
\beta(v)=v=v_{1}+3 v_{2}-3 v_{3}+v_{4}+2 v_{5}-4 v_{6} .
$$

Therefore,

$$
[v]_{\mathbb{B}}=\left[\begin{array}{l}
1 \\
3 \\
-3 \\
1 \\
2 \\
-4
\end{array}\right]=[\beta(v)]_{\mathbb{B}}
$$

and thus

$$
[\beta]_{\mathbb{B}}[v]_{\mathbb{B}}=[\beta(v)]_{\mathbb{B}}
$$

One interesting question to ask will be, can we find a mapping that will transform a refined neutrosophic vector space into a neutrosophic vector space? The answer to this is positive. Since every refined neutrosophic vector space and every neutrosophic vector space are vector spaces, then by relaxing the second axiom in Definition 2.31, the mapping $\phi$ becomes a classical vector space homomorphism which can be use for such transformation.

Proposition 2.44. Let $V\left(I_{1}, I_{2}\right)$ be a weak refined neutrosophic vector space over a field $K$ and let $V(I)$ be a weak neutrosophic vector space over $K$. Let $\phi: V\left(I_{1}, I_{2}\right) \longrightarrow V(I)$ be a mapping defined by

$$
\phi\left(\left(x+y I_{1}+z I_{2}\right)\right)=(x+(y+z) I) \forall\left(x+y I_{1}+z I_{2}\right) \in V\left(I_{1}, I_{2}\right) \text { with } x, y, z \in V .
$$

Then $\phi$ is a linear map.

Proof. 1. $\phi$ is well defined. Suppose $x_{1}+y_{1} I_{1}+z_{1} I_{2}=x_{2}+y_{2} I_{1}+z_{2} I_{2}$ then we that $x_{1}=x_{2}, y_{1}=y_{2}$ and $z_{1}=z_{2}$. So,

$$
\phi\left(\left(x_{1}+y_{1} I_{1}+z_{1} I_{2}\right)\right)=\left(x_{1}+\left(y_{1}+z_{1}\right) I\right)=x_{2}+\left(y_{2}+z_{2}\right) I=\phi\left(x_{2}+y_{2} I_{1}+z_{2} I_{2}\right) .
$$

2. For additivity, suppose $\left(x_{1}+y_{1} I_{1}+z_{1} I_{2}\right),\left(x_{2}+y_{2} I_{1}+z_{2} I_{2}\right) \in V\left(I_{1}, I_{2}\right)$ then

$$
\begin{aligned}
\phi\left(\left(x_{1}+y_{1} I_{1}+z_{1} I_{2}\right)+\left(x_{2}+y_{2} I_{1}+z_{2} I_{2}\right)\right) & =\phi\left(\left(x_{1}+x_{2}\right)+\left(y-1+y_{2}\right) I_{1}+\left(z_{1}+z_{2}\right) I_{2}\right) \\
& =\left(x_{1}+x_{2}\right)+\left(y_{1}+y_{2}+z_{1}+z_{2}\right) I \\
& =\left(x_{1}+x_{2}\right)+\left(\left(y_{1}+z_{1}\right)+\left(y_{2}+z_{2}\right)\right) I \\
& =\left(x_{1}+x_{2}\right)+\left(\left(y_{1}+z_{1}\right) I+\left(y_{2}+z_{2}\right) I\right) \\
& =\left(x_{1}+\left(y_{1}+z_{1}\right) I\right)+\left(x_{2}+\left(y_{2}+z_{2}\right) I\right) \\
& =\phi\left(x_{1}+y_{1} I_{1}+z_{1} I_{2}\right)+\phi\left(x_{2}+y_{2} I_{1}+z_{2} I_{2}\right) .
\end{aligned}
$$


3. For homogeneity, let $\left(x+y I_{1}+z I_{2}\right) \in V\left(I_{1}, I_{2}\right)$ and $k \in K$, then

$$
\begin{aligned}
\phi\left(k\left(x_{1}+y_{1} I_{1}+z_{1} I_{2}\right)\right) & =\phi\left(k x_{1}+k y_{1} I_{1}+k z_{1} I_{2}\right) \\
& =k x_{1}+\left(k y_{1}+k z_{1}\right) I \\
& =k x_{1}+k\left(y_{1}+z_{1}\right) I \\
& =k\left(x_{1}+\left(y_{1}+z_{1}\right) I\right)=k \phi\left(\left(x_{1}+y_{1} I_{1}+z_{1} I_{2}\right)\right) .
\end{aligned}
$$

Hence $\phi$ is a linear map.

Note 3. The kernel of this linear map is given by

$\operatorname{ker} \phi=\left\{\left(x+y I_{1}+z I_{2}\right): \phi\left(\left(x+y I_{1}+z I_{2}\right)\right)=(0+0 I)\right\}$

$=\left\{\left(x+y I_{1}+z I_{2}\right):(x+(y+z) I)=(0+0 I)\right\}$

$=\left\{\left(0+y I_{1}+(-y) I_{2}\right)\right\}$.

1. It can be shown that $\operatorname{ker} \phi$ is a linear subspace of $V\left(I_{1}, I_{2}\right)$.

2. It can also be shown that $(k e r \phi,+) \cong\left(V\left(I_{1}, I_{2}\right),+\right)$.

Proposition 2.45. Let $L_{k}\left(V\left(I_{1}, I_{2}\right), V(I)\right)$ be the set of linear maps from a weak refined neutrosophic vector space $V\left(I_{1}, I_{2}\right)$ over a field $K$ into a weak neutrosophic vector space $V(I)$ over a field $K$. Define addition and scalar multiplication as below;

$$
(\phi+\psi)\left(x+y I_{1}+z I_{2}\right)=\phi\left(\left(x+y I_{1}+z I_{2}\right)\right)+\psi\left(\left(x+y I_{1}+z I_{2}\right)\right)
$$

and for $k \in K$

$$
(k \phi)\left(\left(x+y I_{1}+z I_{2}\right)\right)=k \phi\left(x+y I_{1}+z I_{2}\right) .
$$

Then, it can be shown that $\left(L_{k}\left(V\left(I_{1}, I_{2}\right), V(I)\right),+, \cdot\right)$ is a weak neutrosophic vector space.

Proposition 2.46. Let $\phi \in L_{k}\left(V\left(I_{1}, I_{2}\right), V(I)\right)$ and $\operatorname{dim} V\left(I_{1}, I_{2}\right), \operatorname{dim} V(I)<\infty$.

1. If $\operatorname{dim} V\left(I_{1}, I_{2}\right)>\operatorname{dim} V(I)$, then, no linear map of $V\left(I_{1}, I_{2}\right)$ to $V(I)$ is one to one.

2. If $\operatorname{dim} V\left(I_{1}, I_{2}\right)<\operatorname{dim} V(I)$, then, no linear map of $V\left(I_{1}, I_{2}\right)$ to $V(I)$ is onto.

Proof. 1. Suppose there exist a function $\phi \in L_{k}\left(V\left(I_{1}, I_{2}\right), V(I)\right)$ which is one to one. Then

$$
\operatorname{dim} V\left(I_{1}, I_{2}\right)=\operatorname{dimker} \phi+\operatorname{dim} \operatorname{Im} \phi .
$$

Thus, $\operatorname{dim} V\left(I_{1}, I_{2}\right)=\operatorname{dim} \operatorname{Im} \phi=\operatorname{dim} V(I) \quad(\operatorname{dimker} \phi=0$, since $\phi$ is one to one $)$.

This gives a contradiction. Hence there exist no such function.

2. Suppose there exist a function $\phi \in L_{k}\left(V\left(I_{1}, I_{2}\right), V(I)\right)$ which is onto . Then $\operatorname{Im} \phi=V(I)$. Thus,

$$
\operatorname{dim} V\left(I_{1}, I_{2}\right)=\operatorname{dimker} \phi+\operatorname{dim} \operatorname{Im} \phi
$$

and also

$$
\operatorname{dim} V\left(I_{1}, I_{2}\right) \geq \operatorname{dim} V(I)
$$

Thus

$$
\operatorname{dim} V(I)>\operatorname{dim} V\left(I_{1}, I_{2}\right) \geq \operatorname{dim} V(I) .
$$

This is not possible. Hence there exist no such function.

\section{Conclusion}

This paper studied linear dependence, independence, bases and dimensions of refined neutrosophic vector spaces and presented some of their basic properties. Also, the paper studied refined neutrosophic vector space homomorphisms and established the existence of linear maps between weak refined neutrosophic vector spaces $V\left(I_{1}, I_{2}\right)$ and weak neutrosophic vector spaces $V(I)$. We hope to present more properties of refined neutrosophic vector spaces in our future papers.

\section{Acknowledgment}

The Authors wish to thank the anonymous reviewers for their valuable and useful comments which have lead to the improvement of the paper. 


\section{References}

[1] Abobala, M., On some Special Substructures of Neutrosophic Rings and their Properties, International Journal of Neutrosophic Science, Vol. 4 (2), pp. 72-81, 2020.

[2] Abobala, M., On some Special Substructures of Refined Neutrosophic Rings, International Journal of Neutrosophic Science, Vol. 5 (1), pp. 59-66, 2020.

[3] Abobala, M., Classical Homomorphisms between Refined Neutrosophic Rings and Neutrosophic Rings, International Journal of Neutrosophic Science, Vol. 5 (2), pp. 72-75, 2020.

[4] Adeleke, E.O, Agboola, A.A.A and Smarandache, F. Refined Neutrosophic Rings I, International Journal of Neutrosophic Science (IJNS), Vol. 2(2), pp. 77-81, 2020.

[5] Adeleke, E.O, Agboola, A.A.A and Smarandache, F. Refined Neutrosophic Rings II, International Journal of Neutrosophic Science (IJNS), Vol. 2(2), pp. 89-94, 2020.

[6] Agboola, A.A.A., On Refined Neutrosophic Quotient Groups, International Journal of Neutrosophic Science, vol. 5 (2), pp. 76-82, 2020. DOI:10.5281/zenodo.3828609.

[7] Agboola, A.A.A., Ibrahim, A.M. and Adeleke, E.O, Elementary Examination of NeutroAlgebras and AntiAlgebras Viz-a-Viz the Classical Number Systems, Vol. 4, pp. 16-19, 2020.

[8] Agboola, A.A.A. On Refined Neutrosophic Algebraic Structures, Neutrosophic Sets and Systems, Vol. 10, pp 99-101, 2015.

[9] Agboola, A.A.A., Akinola, A.D. and Oyebola, O.Y., Neutrosophic Rings I, Int. J. of Math. Comb. Vol 4, pp. 1-14, 2011.

[10] Agboola, A.A.A., Adeleke, E.O. and Akinleye, S.A., Neutrosophic Rings II, Int. J. of Math. Comb. Vol. 2, pp 1-8, 2012.

[11] Agboola, A.A.A. Akwu, A.O., and Oyebo, Y.T., Neutrosophic Groups and Neutrosopic Subgroups, Int. J. of Math. Comb. Vol. 3, pp. 1-9, 2012.

[12] Agboola, A.A.A. and Akinleye, S.A., Neutrosophic Vector Spaces, Neutrosophic Sets and Systems Vol. 4, pp. 9-18. 2014.

[13] Atanassov, K., Intuitionistic fuzzy sets, Fuzzy Sets and Systems, Vol. 20, pp. 87-96, 1986.

[14] Bera, T. and Mahapatra,N.K., Introduction to neutrosophic soft groups, Neutrosophic Sets and Systems, Vol. 13, pp, 118-127, 2016, doi.org/10.5281/zenodo.570845.

[15] Bera, T. and Mahapatra, N.K., On neutrosophic normal soft groups, International Journal of Applied and Computational Mathematics., Vol. 3, pp 3047-3066, 2017. DOI 10.1007/s40819-016-0284-2.

[16] Bera, T. and Mahapatra, N.K., On neutrosophic soft rings, OPSEARCH, Vol. 54, pp. 143-167, 2017. DOI 10.1007/ s12597-016-0273-6.

[17] Bera, T and Mahapatra, N. K., On neutrosophic soft linear spaces, Fuzzy Information and Engineering, Vol. 9, pp 299-324, 2017.

[18] Bera, T and Mahapatra, N. K., On neutrosophic soft field, IJMTT, Vol. 56(7), pp. 472-494, 2018.

[19] Deli, I., Broumi, S. and Smarandache, F., On neutrosophic refined sets and their applications in medical diagnosis, Journal of New Theory, Vol. 6, pp. 88-98, 2015.

[20] Ibrahim, M.A., Agboola, A.A.A, Badmus, B.S, Akinleye, S.A., On Refined Neutrosophic Vector Spaces I, (submitted for publication) in International Journal of Neutrosophic Science.

[21] Ibrahim, M.A., Agboola, A.A.A, Adeleke, E.O, Akinleye, S.A., Introduction to Neutrosophic Subtraction Algebra and Neutrosophic Subtraction Semigroup, International Journal of Neutrosophic Science (IJNS), Vol. 2(2), pp. 47-62, 2020.

[22] Ibrahim, M.A., Agboola, A.A.A, Adeleke, E.O, Akinleye, S.A., On Neutrosophic Quadruple Hypervector Spaces, International Journal of Neutrosophic Science (IJNS), Vol. 4(1), pp. 20-35, 2020. 
[23] Liu, P. and Wang, Y., Multiple attribute decision-making method based on single-valued neutrosophic normalized weighted Bonferroni mean, Neural Computing and Applications, Vol. 25 (7-8), pp. 20012010, 2014.

[24] Smarandache, F., Introduction to Neutrosophic Sociology (NeutroSociology), Pons Publishing House/Pons asb1, Quai du Batelage, 5, 1000-Bruxelles, Belgium, 2019.

[25] Smarandache, F. and Pramanik, S., New Neutrosophic Sets via Neutrosophic Topological Spaces, In Operational Research; Eids.; Pons Editions: Brussels, Belgium, Vol. I, pp. 189-209, 2017.

[26] Smarandache, F. and Pramanik, S., Neutrosophic crisp sets via neutrosophic crisp topological spaces, Neutrosophic Sets and Systems, Vol. 13, pp. 96-104, 2016.

[27] Smarandache, F. and Pramanik, S., On Generalized Closed Sets and Generalized PreClosed Sets in Neutrosophic Topological Spaces, Mathematics, Vol. 7(1), pp. 1-12, 2019. DOI:DOI.ORG/10.3390/math7010001.

[28] Smarandache, F., A Unifying Field in Logics: Neutrosophic Logic, Neutrosophy, Neutrosophic Set, Neutrosophic Probability, American Research Press, Rehoboth, 2003.

[29] Smarandache, F., n-Valued Refined Neutrosophic Logic and Its Applications in Physics, Progress in Physics, Vol. 4, pp. 143-146, 2013.

[30] Smarandache, F., (T,I,F)- Neutrosophic Structures, Neutrosophic Sets and Systems, Vol.8, pp.3-10, 2015.

[31] Vasantha Kandasamy, W.B and Smarandache,F., Basic Neutrosophic Algebraic Structures and Their Applications to Fuzzy and Neutrosophic Models, Hexis, Church Rock, (2004), http://fs.unm.edu/ScienceLibrary.htm

[32] Vasantha Kandasamy, W.B. and Florentin Smarandache, Some Neutrosophic Algebraic Structures and Neutrosophic N-Algebraic Structures, Hexis, Phoenix, Arizona, (2006), http://fs.unm.edu/ScienceLibrary.htm

[33] Vasantha Kandasamy, W.B., Neutrosophic Rings, Hexis, Phoenix, Arizona,(2006) http://fs.unm.edu/ScienceLibrary.htm

[34] Vasantha Kandasamy, W.B., Ilanthenral Kandasamy and Smarandache, F., Neutrosophic Quadruple Vector Spaces and Their Properties, Mathematics, Vol. 7, pp. 758-765, 2019. http://fs.unm.edu/ScienceLibrary.htm

[35] Wadei Al-Omeri and Smarandache, F., New Neutrosophic Set via Neutrosophic Topological Spaces. Excerpt from Neutrosophic Operation Research Vol I, Pons Editions: Brussels, Belgium, pp. 189-209, 2017.

[36] Wadei Al-Omeri, Neutrosophic crisp Sets Via Neutrosophic crisp Topological Spaces, Neutrosophic Set and Systems Vol 13, pp 96- 104, 2016.

[37] Wadei Al-Omeri and Saeid Jafari, On Generalized Closed Sets and Generalized Pre-Closed Sets in Neutrosophic Topological Spaces, Mathematics, Vol 7, pp 1- 12, 2019. Doi: doi.org/10.3390/math/7010001.

[38] Zadeh, L.A., Fuzzy Sets, Information and Control, Vol. 8, pp. 338-353, 1965. 